\title{
Plant Chromatin Catches the Sun
}

\author{
Clara Bourbousse ${ }^{1 *}$, Fredy Barneche ${ }^{1 *}$ and Christophe Laloi $^{2}$ \\ 1 Institut de Biologie de l'Ecole Normale Supérieure (IBENS), Ecole Normale Supérieure, CNRS, INSERM, Université PSL, \\ Paris, France, ${ }^{2}$ Aix Marseille Univ, CEA, CNRS, BIAM, Luminy Génétique et Biophysique des Plantes, Marseille, France
}

Plants use solar radiation as energy source for photosynthesis. They also take advantage of the information provided by the varying properties of sunlight, such as wavelength, orientation, and periodicity, to trigger physiological and developmental adaptations to a changing environment. After more than a century of research efforts in plant photobiology, multiple light signaling pathways converging onto chromatin-based mechanisms have now been identified, which in some instances play critical roles in plant phenotypic plasticity. In addition to locus-specific changes linked to transcription regulation, light signals impact higher-order chromatin organization. Here, we summarize current

OPEN ACCESS

Edited by:

Paula Casati,

CONICET Center for Photosynthetic and Biochemical Studies (CEFOBI),

Argentina

Reviewed by:

Giorgio Perrella,

ENEA-Centro Ricerche Trisaia, Italy

María Sol Gomez,

CONICET Center for Photosynthetic and Biochemical Studies (CEFOBI),

Argentina

*Correspondence: Clara Bourbousse bourbous@biologie.ens.fr Fredy Barneche barneche@ens.fr

Specialty section: This article was submitted to

Plant Abiotic Stress,

a section of the journal

Frontiers in Plant Science

Received: 31 July 2019 Accepted: 09 December 2019 Published: 24 January 2020

Citation:

Bourbousse C, Barneche F and Laloi C (2020) Plant Chromatin Catches the Sun.

Front. Plant Sci. 10:1728. doi: 10.3389/fpls.2019.01728 knowledge on how light can affect the global composition and the spatial distribution of chromatin domains. We introduce emerging questions on the functional links between light signaling and the epigenome, and further discuss how different chromatin regulatory layers may interconnect during plant adaptive responses to light.

Keywords: plants, light, photoperception, nucleus, chromatin, 3D genome

\section{INTRODUCTION}

From the early studies on daylength and flowering time by Julien Tournois and Georg Albrecht Klebs before the first World War (reviewed in Sage, 1992), a century of research efforts in plant photobiology has permitted the identification of sensory mechanisms that allow plants to cope with fluctuating light conditions over their lifetime (Chory, 2010). Intensity, direction, and spectral composition of light constitute crucial sources of spatio-temporal information for a plant about its environment, for example about photoperiod, season, and presence of neighboring or shadeproducing competitors. In addition, while being essential for plant growth and development light can also be harmful at high intensities. Overloading the photosynthetic electron transport chain notably leads to the production of reactive oxygen species (ROS), which can cause irreversible damage to cellular components. Exposure to strong sunlight further exposes plants to the detrimental effects of ultraviolet (UV) radiations on photosynthetic activity, cell integrity, and genome stability. Balancing the needs of photon harvesting for photosynthesis with photoprotection and developmental responses to changing light conditions is therefore at the nexus of plant fitness (Li et al., 2009; Croce and van Amerongen, 2014; Rochaix, 2014; Demarsy et al., 2018).

Plants can integrate light signals through a set of cytosolic or nuclear photoreceptors (Figure 1). Photoreceptors allow sensing specific solar wavelengths through an associated chromophore (red and far-red light by phytochromes, blue light by cryptochromes, phototropins, and Zeitlupe) or through tryptophan residues in the case of UV RESISTANCE LOCUS 8 (UVR8) (Reviewed in Galvão and Fankhauser, 2015; Demarsy et al., 2018). When excited by photons, photoreceptors initiate complex regulatory cascades ultimately controlling the expression of vast repertoires of light-responsive genes. In the Arabiopsis model plant species, key signaling components are the 


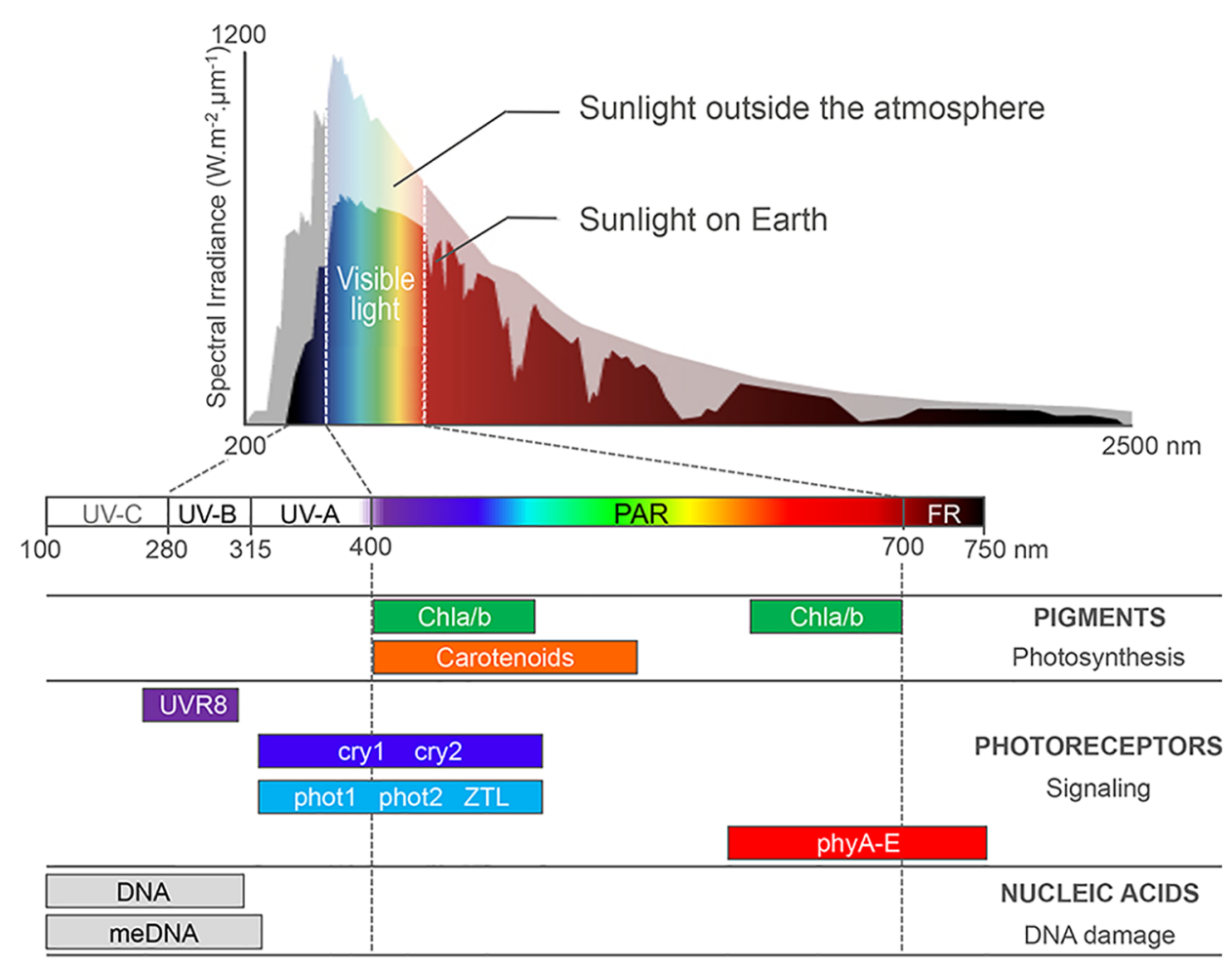

FIGURE 1 | Sunlight absorption by Arabidopsis cell compounds. Most sunlight visible wavelengths penetrate the atmosphere, whereas harmful UV-C and part of UV-B/A are absorbed by ozone in the atmosphere. Photosynthetic pigments, such as the carotenoids and chlorophyll a and b (Chla/b) harvest photons in the 400- to 700-nm waveband, which correspond to photosynthetically active radiations (PAR). A battery of nuclear photoreceptors sensitive to blue, red, far-red, or UV-B light trigger specific adaptations of plant development and metabolism to the local environment. Finally, aromatic DNA bases and methylated cytosines absorb light in the UV waveband, enhancing DNA susceptibility to genotoxic damages and mutations. Adapted from Fondriest Environmental, Inc., (2014) and from Kami et al. (2010).

photomorphogenesis master repressors DE-ETIOLATED-1 (DET1) and CONSTITUTIVE PHOTOMORPHOGENIC 1 (COP1) proteins that control the stability of photoreceptors and transcription factors, such as the PHYTOCHROME INTERACTING FACTORS (PIFs) and ELONGATED HYPOCOTYL 5 (HY5) (reviewed in Casal, 2013; Galvão and Fankhauser, 2015; Seluzicki et al., 2017). Among these factors, the discovery of DET1 association to nucleosomes has constituted a first molecular function linking light signaling and chromatin (Benvenuto et al., 2002). The precise impact of this evolutionarily conserved factor and of related proteins on chromatin has just started to be unveiled (reviewed in Fonseca and Rubio, 2019). More generally, functional genetic studies have now unveiled the importance of chromatin-level control of gene expression in plant adaptive responses to light conditions. Under high light irradiance, chromatin responses to damaging doses of UV or to stressful plastid activity further contribute to preserving genome activity and stability.

Multiple histone post-translational modifications and chromatin-bound factors act sequentially or in combination to fine-tune the transcriptional activity of light-responsive genes. A first set of genome-wide studies allowed identifying a range of histone marks and nucleosome organization dynamics that contribute to efficient gene expression regulation during Arabidopsis cell adaptation to light (Charron et al., 2009; Bourbousse et al., 2012; Sullivan et al., 2014; Pass et al., 2017). At a larger scale, cytological approaches have identified dynamic changes of chromosomal domains and of specific genes into the nuclear space in response to light signaling. The corresponding pathways converging onto specific chromatin loci or regulating either abundance or activity of chromatin machineries have also started to emerge. Here we report the main advances in understanding the chromatin mechanisms used by plants to cope with solar radiation.

\section{LIGHT PERCEPTION TRIGGERS MASSIVE NUCLEAR REORGANIZATION}

\section{Heterochromatin Dynamics}

The monitoring of nuclear architecture variations during Arabidopsis post-embryonic cotyledon development has unveiled a serial reorganization process during germination and seedling establishment that relies on light perception (Figure 2). In dry seeds, cotyledon nuclei are extremely small while heterochromatic domains, mostly constituted of 


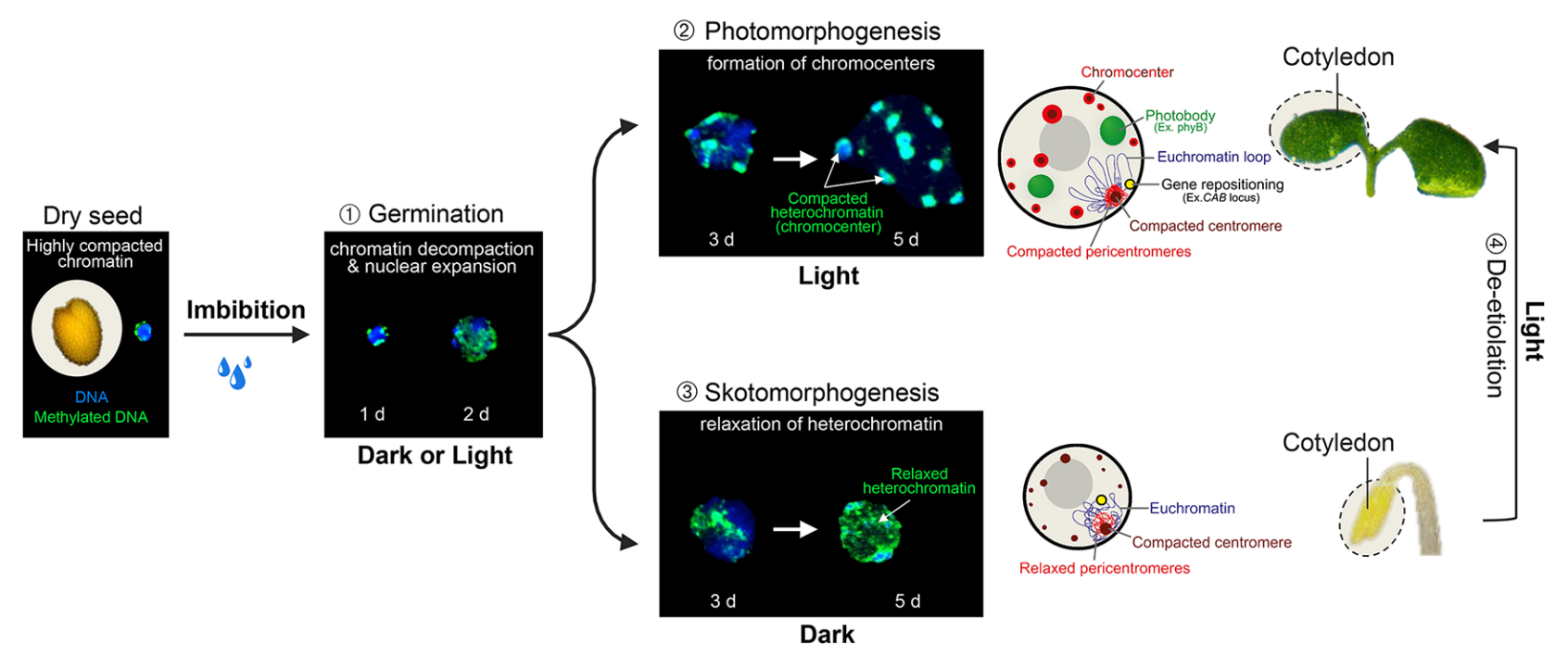

FIGURE 2 | Light-dependent nuclear organization dynamics in cotyledons. During the first 2 days of germination, seed imbibition initiates nuclear expansion and heterochromatin decondensation, as shown by the dispersed signal of methylated DNA immunolabeling in both dark and light conditions (Phase 1). From the third day of germination, light perception triggers further nuclear expansion and the re-compaction of pericentromeric domains within cytologically observable chromocenters (Phase 2), whereas in the absence of light, heterochromatin is further decondensed (Phase 3). Skotomorphogenic nuclear phenotypes can rapidly be converted to a photomorphogenic-type organization during cotyledon de-etiolation (Phase 4). Cotyledon de-etiolation also involves the relocation of light-inducible genes to the nuclear periphery (such as the CAB locus), and the aggregation of photoreceptors and downstream signaling components, such as phyB, COP1, PIFs, and TZP into sub-nuclear speckles referred to as photobodies. d; days post-imbibition.

centromeres, pericentromeres, and silent repeat elements, such as Transposable Elements (TEs) (reviewed in Simon et al., 2015), are highly compacted in two to four subnuclear foci. This peculiar nuclear phenotype may constitute a protective mechanism to desiccation (van Zanten et al., 2011). Upon seed imbibition, a first transition involves an extensive relaxation of heterochromatin and moderate nuclear expansion (Figure 2; Phase (1), which is achieved under either dark or light conditions (Bourbousse et al., 2015). A second step occurring 3 days after imbibition involves additional increase of nuclear size and the recompaction of dispersed heterochromatin regions into 8 to 10 sub-nuclear regions referred to as chromocenters (Mathieu et al., 2003; Douet et al., 2008; van Zanten et al., 2011), a typical organizational scheme observed in most cell types of Arabidopsis adult plants (Fransz et al., 2002). This second step is dependent on light signaling (Phase (2) as the formation of conspicuous chromocenters relies on blue light sensing by cryptochromes. In the absence of light, heterochromatin compaction is both inhibited by the DET1 and COP1 signal integrators (Phase (3). This arrest is rapidly released during de-etiolation (Phase (4)) suggesting that nuclear development is poised prior to the acquisition of phototrophy (Bourbousse et al., 2015).

Upon transition to adult developmental stages, Arabidopsis nuclear architecture also undergoes several reorganizational events driven by light signals (reviewed in van Zanten et al., 2012). This is notably the case in rosette leaf mesophyll cells during the transition from vegetative to reproductive growth. Leaves integrate endogenous and environmental signals to trigger the conversion of apical shoot meristems into flowering meristems. Remarkably, in leaf mesophyll nuclei, most chromocenters undergo a significant disruption a few days before bolting with the dispersion of $5 \mathrm{~S}$ rDNA and pericentromeric repeats (Tessadori et al., 2007). This transient phenomenon is developmentally controlled, as chromocenters are restored 3 days after bolting. The decompaction process relies on cry2-mediated blue light sensing, which therefore has an opposite effect during this transition than during cotyledon morphogenesis where it triggers chromocenter formation. Flowering time is delayed when CRY2 function is impaired (Guo et al., 1998), suggesting that a large-scale chromatin decompaction episode is linked to the flowering transition (Tessadori et al., 2007).

A link between light fluence rate and chromatin spatial organization is further suggested by the observation that exposure to low light intensity for a few days triggers the dispersion of most chromocenters in rosette mesophyll cells (Tessadori et al., 2009; van Zanten et al., 2010b; van Zanten et al., 2010a). Again, this event is reversible and relies on light signaling through cry2 and phyB photoreceptors (reviewed in van Zanten et al., 2010a). Interestingly, this mechanistic link might be adaptive in the wild, as population genetics analysis of Arabidopsis thaliana accessions identified a correlation between latitude of origin, PHYB amino acid sequence polymorphisms and heterochromatin compaction levels in mesophyll cells (Tessadori et al., 2009; Snoek et al., 2017). Heterochromatin compaction might therefore be an integral part of the plant 
response to short-term fluctuating light as well as to the annual light radiation regime sensed by phytochrome photoreceptors having evolved different sensitivities to local sunlight conditions.

\section{Light Regulation of Sub-Nuclear Micro- Environments}

In addition to heterochromatin dynamics, Arabidopsis nuclei are subjected to several reorganization events in response to light, which directly impact euchromatic loci, and therefore also protein-coding genes. A landmark study using single-locus cytological experiments unveiled that several light-inducible genes, such as $C A B$ (chlorophyll a/b-binding proteins) and RBCS (Rubisco small subunit), are re-positioned from the interior to the periphery of nuclei during Arabidopsis cotyledon de-etiolation (Figure 2 Phase (4)). In this context, the physical movement of genes might hypothetically facilitate mRNA export through nuclear pore complexes. Being promoted by phytochrome-dependent red light sensing and inhibited by COP1, DET1, and PIFs, this process appears to be controlled by light signaling (Feng et al., 2014a). Still, as for heterochromatin condensation dynamics, the functional implications of light-dependent gene motion on transcription or on mRNA processing and export remain to be assessed. This could possibly be achieved by artificially tethering a given locus to distinct sub-nuclear compartments and monitoring its resulting transcriptional activity.

Gene repositioning is a dynamic process that echoes the rapid relocalization of light signaling components within conspicuous nuclear speckles, a process observed in both dicotyledonous and monocotyledonous plants when exposed to light. More precisely, upon photoexcitation most nuclear-localized photoreceptors, including the five phytochromes phyA-E, UVR8, cry2, and possibly also cry1, concentrate into nuclear bodies referred to as "photobodies." The function and composition of the diverse types of photobodies remain elusive. They have been envisioned as either subnuclear sites of light signaling, of protein degradation or as transcription hubs (Van Buskirk et al., 2012), three functions that might be dynamically interconnected. The colocalization of multiple transcriptional regulators such as COP1, PIFs, and TANDEM ZINC-KNUCKLE PLUS3 (TZP) within nuclear microenvironments supports this scenario (reviewed in Van Buskirk et al., 2012; Perrella and Kaiserli, 2016).

\section{Other Types of Light-Induced Nuclear Reorganization Events}

Light perception directly influences nuclear DNA content, a feature with potential high impact on nuclear organization and activity. Low light intensity dampens endoreduplication, for example maintaining low ploidy levels in Arabidopsis leaves (Cookson et al., 2006). A similar trend is at play in etiolated hypocotyls where darkness triggers an additional endocycle (Gendreau et al., 1997) while, conversely, cotyledon deetiolation engages an increase of DNA ploidy levels (LopezJuez et al., 2008). This contrast illustrates the organ-specificity of genome responses to light. DNA content and nuclear volume being positively correlated in angiosperms (Jovtchev et al., 2006), endoreduplication presumably allows for a rapid increase in nucleus and cell size, which is promoted in hypocotyls during seedling etiolation.

There is evidence that ploidy may then influence nuclear organization and gene expression during photomorphogenesis. Firstly, the sub-nuclear positions of chromocenters tend to vary with ploidy level and nuclear volume in Arabidopsis pavement cells, highly endoreduplicated nuclei having more internal chromocenters (Poulet et al., 2017). Second, nuclei with elevated ploidy levels display lowly condensed heterochromatin and low chromatid cohesion (Schubert et al., 2012). Of note, endopolyploidy potentially also influences gene expression by gene dosage effects (Doyle and Coate, 2018). Finally, exposure to high light intensity appears to stimulate endopolyploidy in epidermal pavement cells of Arabidopsis and Phaseolus (Kinoshita et al., 2008). Increasing genome copy number may in this case allow to cope better with the mutagenic effects of UV light exposure (Gegas et al., 2014).

Plant responses to damaging solar irradiations constitute another type of light-related process of particular interest, especially if considering not only the deleterious effect of UV on DNA but also the plant capacity to use UV-B perception to modulate photomorphogenesis (Jenkins, 2017; Yin and Ulm, 2017). Despite the presence of sunscreen components and of efficient DNA-repair pathways induced by light, UV radiations have now been shown to impact plant epigenomes in multiple ways (reviewed in Kimura and Sakaguchi, 2006). The role played by chromatin processes in plant DNA repair after UV damage and the mechanisms of chromatin restoration after the completion of repair have been detailed elsewhere (see for example Donà and Mittelsten Scheid, 2015; Molinier, 2017). On a different note, damaging doses of UV-B increase the appearance of heritable DNA mutations with higher frequency on 5-mC cytosines, especially in TC sequence contexts. This highlights the influence of the epigenome on genome integrity (Willing et al., 2016). This effect might relate to the high UV-B absorbance by methylated pyrimidines, which potentially enhances the formation of photoproduct dimers (reviewed in Molinier, 2017). Vice versa, UV-C induced photolesions have recently been shown to be a source of DNA methylation changes in heterochromatin (e.g., over pericentromeric TEs) (Graindorge et al., 2019). These findings unveil intricate links between DNA repair factors and the accurate maintenance of epigenome integrity.

UV exposure has further been shown to trigger large-scale heterochromatin rearrangements in Arabidopsis (Graindorge et al., 2019). Still, as for blue-light induced chromocenter relaxation, their functional meaning remains unappreciated. In mammals, heterochromatic regions appear to be particularly prone to DNA mutations, possibly representing a peripheral umbrella to harmful radiation for genes located in the euchromatic interior of the nuclei (Schuster-Böckler and Lehner, 2012; Takata et al., 2013; Smith et al., 2017). Future studies might assess whether different plant heterochromatin 
organizational patterns or variations in genome topology differ in mutational rates.

\section{LIGHT SIGNALING MODULATES THE EPIGENOME LANDSCAPE}

\section{Light Signaling Controls the Abundance of Chromatin Modifiers and Homeostasis of Histone Marks}

Landmark work by the Gray's laboratory allowed linking histone acetylation with nucleosome occupancy and transcription at the PetE photosynthetic gene promoter in green and etiolated shoots of pea seedlings (Chua et al., 2001; Chua et al., 2003). From then, an ever increasing number of studies have dissected the role played by chromatin modifying activities in light-regulated gene expression (reviewed in Li et al., 2012; Barneche et al., 2014; Perrella and Kaiserli, 2016; Xiao et al., 2017; Duarte-Aké, 2019). Accordingly, several mutants affected in the deposition or the removal of histone post-translational modifications display photomorphogenesis-related phenotypes. For example, Arabidopsis mutants in the GCN5 and TAF1 histone acetyltransferases (HAT) display shorter hypocotyls and lower expression of light-inducible genes, and so, are hyposensitive to light. On the contrary, knocking-out HD1 or HDA15 histone deacetylase (HDAC) genes trigger exaggerated inhibition of hypocotyl elongation and other light hypersensitivity phenotypes (Benhamed et al., 2006; Liu et al., 2013). Histone acetyltransferases such as HAF1 and HAC1 are also at play to control gene expression and adaptive developmental responses to UV-B signaling (e.g., plant growth inhibition, flowering time acceleration) (Fina et al., 2017). Hence, photomorphogenesis appears to involve a tight balance between the opposing activities of histone acetyltransferases and deacetylases. In line with these findings, DET1 hypomorphic mutants display a wide deregulation of histone acetylation levels (Nassrallah et al., 2018). A gene-specific regulatory mechanism involves the light-controlled nucleo-cytoplasmic partitioning of the HDA15 histone deacetylase and its physical association with the transcription factors HY5, PIF3, and NUCLEAR FACTOR-Y C (NF-YCs) in the nucleus (Alinsug et al., 2012; Liu et al., 2013; Tang et al., 2017; Zhao et al., 2019) (Figure 3A). Finally, a forward genetic screen aimed at identifying novel regulators of carbon and light signaling identified SDG8, a histone methyltransferase responsible for maintaining high levels of H3K36me3 towards 3 '-end of many photosynthetic and metabolic genes ( $\mathrm{Li}$ et al., 2015).

Other than histone modifiers, the regulation of chromatin functional states in response to light also involves chromatin remodelers. By displacing nucleosomes or facilitating histone exchange, these factors can modulate nucleosome positioning and histone-DNA interactions, thereby influencing local access to transcriptional machineries [reviewed in (Han et al., 2015)]. The importance of such regulatory mechanisms in plant adaptations to light is illustrated by the hundreds of photodynamic DNase/MNase Hypersensitive Sites (DHS) identified in Arabidopsis during seedling de-etiolation or during cell adaptation to light, which typically lie next to lightresponsive genes (Sullivan et al., 2014; Pass et al., 2017). A member of the CHD3 family of chromatin remodelers, PICKLE (also referred to as Enhanced Photomorphogenesis 1) has been identified in a forward genetic screen for negative regulators of photomorphogenesis (Figure 3B). PICKLE accumulates and associates with the promoter of hypocotyl elongation promoting genes in darkness, thereby preventing deposition of the repressive Polycomb-associated histone mark H3K27me3 (Jing et al., 2013). PICKLE interacts with the master regulatory transcription factors HY5, PIF3, and BZR1, which target its chromatin remodeling activity to light-responsive loci (Jing et al., 2013; Zhang et al., 2014). Similarly, two subunits of the SWI/SNF-type ATP-dependent family of chromatin remodelers also show a light-dependent accumulation. Following exposure to light, the accessory subunit BAF60 (also named $\mathrm{CHC1}$ or SWP73B) accumulates and is recruited to the promoters of genes regulating hypocotyl elongation, possibly antagonizing PIF activity through competitive binding onto Gbox motifs (Jégu et al., 2017). Reciprocally, abundance of the BRAHMA SWI2/SNF2-type ATPase is lowered upon exposure to light, thereby promoting expression of chlorophyll biosynthetic genes. The PIF1 transcription factor physically associates with BRAHMA, again mediating a cis-regulatory gene repression mechanism (Zhang et al., 2017). Taken together, these findings exemplify how light signaling pathways target distinct chromatin remodeling machineries to operate a tight and fine tuning of the light-responsive gene repertoire. They also unveiled how light perception influences the availability of chromatin machineries, a regulatory mechanism potentially mediating large effects over the epigenome.

Correspondingly, bulk levels of histone marks are themselves controlled during cell specification, a critical process in human cancer (Berger, 2000; Jeusset and McManus, 2019) that emerged in plant systems first in the context of gamete formation (Baroux et al., 2007; She et al., 2013; He et al., 2019). For example, abundance of linker histone variant $\mathrm{H} 1.3$ and monoubiquitinated histone $\mathrm{H} 2 \mathrm{~B}(\mathrm{H} 2 \mathrm{Bub})$ is globally regulated in Arabidopsis seedlings in response to light (Rutowicz et al., 2015; Nassrallah et al., 2018). While the function of H1.3 gene induction by low light remains unclear, establishment of the $\mathrm{H} 2 \mathrm{Bub}$ mark is thought to facilitate RNA Polymerase II processivity across nucleosomes, notably through cotranscriptional cycles of histone $\mathrm{H} 2 \mathrm{~B}$ ubiquitination/deubiquitination (Henry et al., 2003). In Arabidopsis, H2Bub deposition is necessary in cis for efficient inducibility of hundreds genes during de-etiolation (Bourbousse et al., 2012). Recent work further unveiled that the C3D complex (made of COP10, DET1, DDB1, and DDA1) associates with an H2Bub deubiquitination module (DUBm) that regulates $\mathrm{H} 2 \mathrm{Bub}$ levels over most, if not all, Arabidopsis genes (Figure 3C). Ubiquitinmediated proteasomal degradation of this DUBm in response to light appears to allow for a tight control of $\mathrm{H} 2 \mathrm{Bub}$ homeostasis (Nassrallah et al., 2018). Low H2Bub abundance 


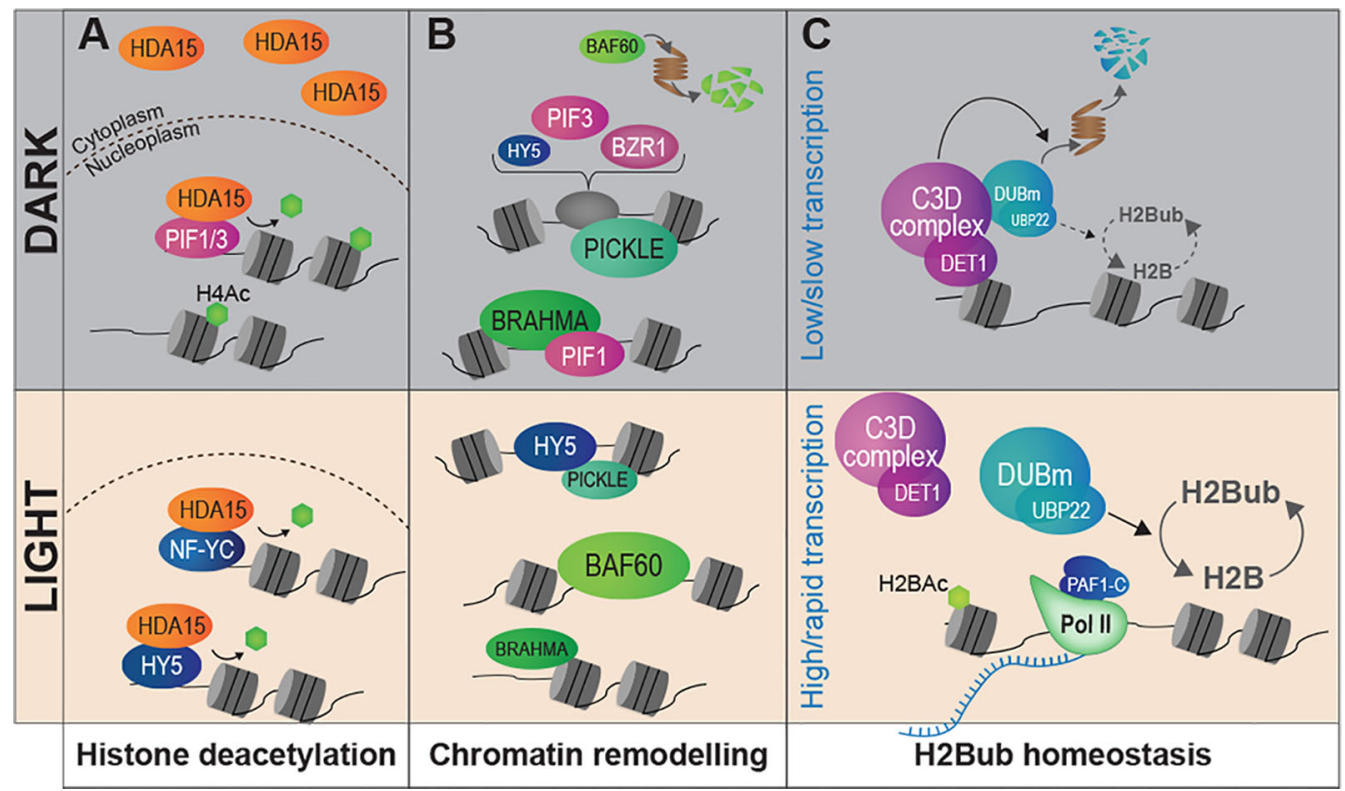

FIGURE 3 | Light signaling controls the abundance of chromatin modifiers and the homeostasis of histone marks. (A) Light signaling modulates the sub-cellular partitioning of HDA15, a histone deacetylase that tends to relocate to the nucleus in response to light. In darkness, low amounts of HDA15 retained in the nucleus could target specific light-induced genes through physical association with PIF3. Under light conditions the bulk of HDA15 proteins locate in the nucleus and can associate with NF-YC and HY5. (B) The availability of chromatin remodeler subunits is influenced by light conditions. PICKLE and BRAHMA are abundant in darkness whereas BAF60 is expressed and stabilized under light where it may antagonize PIF4 activity. Direct interactions with key TFs of the light-signaling pathway have been demonstrated for PICKLE (with HY5 and PIF3) and for BRAHMA (with PIF1). (C) The co-transcriptional H2Bub histone mark is more abundant over most genes in light than dark-grown seedlings. The C3D complex associates to non-acetylated histone $\mathrm{H} 2 \mathrm{~B}$ and controls the stability of a de-ubiquitination module (DUBm), notably through targeted ubiquitin-mediated proteolytic degradation in darkness. Modulation of H2Bub homeostasis over the epigenome may be related to dampened RNA Pol II activity in cotyledon nuclei in darkness.

over most genes during skotomorphogenesis might hypothetically be linked to the globally low RNA Pol II activity observed in etiolated cotyledons (Bourbousse et al., 2015). These studies echo the recent report of low PolymeraseAssociated-1 (PAF1) complex subunits expression and slow RNA Pol II elongation in dark-adapted Arabidopsis plants (Godoy Herz et al., 2019).

In brief, besides gene-specific targeting mechanisms and spatial rearrangements of chromatin domains, light appears to modulate the homeostasis of several histone marks, notably through a tight control of chromatin modifiers' availability. Light therefore not only reshuffles chromatin states at induced and repressed genes but also deeply modify chromatin composition as a whole. Such genome-level chromatin changes might enable an adjustment of the chromatin landscape to the cell transcriptional status (Figure 3C).

\section{Direct Paths From Light Signals to Chromatin States}

As presented in the previous sections, chromatin modifiers and remodelers can be targeted to specific loci by the means of lightcontrolled transcription factors thereby constituting direct signaling pathways. Noteworthy, phytochromes also interact with PIFs (Ni et al., 1998; Leivar and Quail, 2011), cryptochromes with CIB1 and PIF4 (Liu et al., 2008; Ma et al., 2016; Pedmale et al., 2016) and UVR8 can interact with BES1 (BRI1-EMS-SUPPRESSOR1) and BIM1 (BES1-INTERACTING MYC-LIKE 1) (Liang et al., 2018). Profiling of the PHYA, PHYB, CRY1, and CRY2 association to chromatin further shed light on the genomic distribution of photoreceptors in seedlings exposed to either white, blue, far-red, or low blue-light conditions, respectively (Chen et al., 2014; Jung et al., 2016; Ma et al., 2016; Pedmale et al., 2016). The reported binding sites largely overlap with those of light-regulated transcription factors suggesting that photoreceptors bind chromatin indirectly via protein-protein interactions. Yet, following the first cytological observations of Arabidopsis CRY2 association to mitotic chromosomes (Cutler et al., 2000), this protein has been reported to display intrinsic DNA-binding properties (Yang et al., 2018). Finally, based on its sequence similarity to human RCC1 (REGULATOR OF CHROMATIN CONDENSATION 1) and on nucleosome binding assays, the UV-B photoreceptor UVR8 has long been hypothesized to act directly on chromatin (Brown et al., 2005; Cloix and Jenkins, 2008; Favory et al., 2009; Binkert et al., 2016). The functional meanings of UVR8 in vitro association to chromatin findings remain to be established. These physical associations imply the existence of extremely short paths linking light sensing and molecular implementation in the epigenome. 
In addition to photoreceptors, other nuclear light signaling components have the capacity to associate with histones and can therefore be considered themselves as chromatin factors. Noteworthy, DET1 displays high affinity for non-acetylated histone $\mathrm{H} 2 \mathrm{~B}$ both in vitro and in vivo and therefore potentially associates to poorly transcribing genes (Benvenuto et al., 2002). Accordingly, Arabidopsis DET1 triggers efficient transcription inhibition when targeted to reporter genes in plants or in a yeast heterologous system (Maxwell et al., 2003; Lau et al., 2011). Still, it remains unclear whether DET1 repressive activity relies directly on targeted degradation of the H2Bub DUBm at regulated chromatin loci in cis (Nassrallah et al., 2018) and whether that process is targeted by DET1-associated transcription factors such as HY5 and PIFs (Osterlund et al., 2000; Dong et al., 2014).

Being located close to chromatin, photo-excited photoreceptors or downstream light signaling components therefore have the potential to couple light sensing with a functional rewiring of chromatin in situ. Such a direct coupling between light sensing and chromatin may allow for rapid transcriptional reprogramming, thereby resembling metazoan nuclear receptors that combine transcription factor and ligandbinding activities (Perissi and Rosenfeld, 2005). Additionally, the lack of a signal amplification cascade may prevent spontaneous activations that are usually avoided by negative feedback regulations (Kiel et al., 2010).

\section{Retrograde and Metabolic Signals to Chromatin?}

Besides the direct perception of light by nuclear photoreceptors, photosynthetic light harvesting might also impact plant nuclear organization and chromatin states through more indirect paths. Photon harvesting by photosystem antennae directly influences the energetic and redox status of plant cells (Eberhard et al., 2008). Plastids can therefore act as environmental sensors and communicate information about ambient light conditions to the nucleus, notably through retrograde signaling mediated by metabolite and protein signals (Chan et al., 2016). The extent to which retrograde signaling impacts on epigenome organization and function remains largely unexplored. Still, identification of a nuclear topoisomerase VI (Topo VI; Yin et al., 2002) as being essential for the activation of nuclear genes in response to a plastid-derived photo-oxidative stress exemplifies the importance of chromatin-level control of nuclear responses to high light (Šimková et al., 2012). Loss of Topo VI subunits leads to heterochromatin disorganization (Kirik et al., 2007), as observed under dark and low light conditions. In addition, several chromatin modifying enzymes catalytic activity directly relies on the availability of substrate metabolites, such as acetyl-CoA, S-adenosyl methionine (SAM) and adenosine tri-phosphate for histone acetyltransferases, histone/DNA methyltransferases and chromatin remodeling ATPases, respectively. In mammals, direct coupling between redox homeostasis and chromatin state involves the histone deacetylases from the Sirtuin family (reviewed in Suganuma and Workman, 2018), whose activity requires NAD+ as a cofactor. In plants as well, an Arabidopsis Sirtuin-like protein, AtSIRT1, has recently been involved in stress tolerance and metabolic regulations (Liu et al., 2017a). Further investigation is needed to identify how metabolic and redox cellular status impact on chromatin organization and function. In particular, much remains to be explored on the influence of plastid biogenesis and photosynthesis on the chromatin landscape during cell adaptations to environmental changes.

\section{LINKING VARIATIONS IN LOCAL CHROMATIN STATUS WITH 3D RE- ORGANIZATION EVENTS}

Temporal correlations between light-induced gene motion and chromocenter formation suggested that genome $3 \mathrm{D}$ reorganization is an integral part of Arabidopsis de-etiolation (Barneche et al., 2014; Kaiserli et al., 2018). Gene re-positioning or photobody formation could be facilitated when chromatin is globally decompacted. In this scenario, a relaxed chromatin status might retain high flexibility to specify different genome topologies in response to environmental conditions that plant may predictably face. Accordingly, heterochromatin decompaction is an evolutionarily conserved feature common to undifferentiated eukaryotic cells, as shown for mammalian embryonic stem cells or plant protoplasts (Probst and Almouzni, 2011). Dynamic control of the chromatin association of light signaling components might not only allow for targeted gene regulation but potentially also modulate nuclear organization.

As described above, many light signaling components and transcription factors relocate within photobodies upon photoreceptors excitation. Concentrating light transcriptional regulators within a small number of nuclear micro-domains might functionally organize genes that are similarly regulated by light but distantly encoded in the genome. In the absence of clear evidence for "transcription factory" foci in plant nuclei, determining whether photobodies actually correspond to transcriptional foci and whether they contain light-regulated genes might help better understanding the spatial regulation of transcription in plant systems.

Still, although higher-order heterochromatin dynamics usually coincide with genome expression reprogramming events, we lack information on the extent to which chromocenter dispersion in the nucleoplasm actually relies, at the molecular level, on physical changes in the structure or composition of heterochromatic domains. We also do not know how heterochromatin relaxation events functionally impact on euchromatic domains and in particular on protein-coding genes (Feng et al., 2014b; Liu et al., 2017b). Generating high-resolution maps of genome topological variations in response to changing light regimes therefore represents a critical step to characterize gene motion in cell types with distinct light-response specificities. The implementation of chromosomal conformation capture derived approaches (3C; Bickmore and van Steensel, 2013), super-resolution microscopy or adapted chromatin profiling techniques (Re-ChIP, ChIA-PET, etc) 
applied to peripheral components as in (Bi et al., 2017) or to specific nuclear regions will surely boost these research efforts in the coming years.

\section{PERSPECTIVES}

Recent findings unveil that cell differentiation during many plant adaptive responses to light involves large and rapid rearrangements of the epigenome landscape and of genome topology in photosynthetic cells. Still, the functional impact of chromocenter formation, photobody dynamics, and gene repositioning on transcription remain speculative in most instances. It is also currently unclear whether heterochromatin reorganization and gene motion are mechanistically or functionally interconnected. Regarding Arabidopsis deetiolation case studies, it remains to be assessed whether nuclear architectural adaptations occurring upon the initial seedling adaptation to light are linked to the dramatic changes in metabolic activity following chloroplast biogenesis, to cell cycle control, to the process of cell differentiation itself or more directly to the cell transcriptional regime. Vice versa, chromatin architecture dynamics may also impact photon transmission through plant cell nuclei. An inverted nuclear organization with heterochromatin being concentrated toward the interior of the nucleus indeed constitutes a crucial adaptation of rod cells for nocturnal lifestyle, thereby allowing a more efficient channeling of light (Solovei et al., 2009; Solovei et al., 2013). Whether light-regulated heterochromatin rearrangements can allow for biophysical adaptations favoring photon penetration into certain plant cell layers is largely unexplored. Finally, the potential capacity of light to trigger long-term somatic adaptive

\section{REFERENCES}

Alinsug, M. V., Chen, F. F., Luo, M., Tai, R., Jiang, L., and Wu, K. (2012). Subcellular localization of class II HDAs in Arabidopsis thaliana: nucleocytoplasmic shuttling of HDA15 is driven by Light. PloS One 7, e30846. doi: 10.1371/journal.pone.0030846

Barneche, F., Malapeira, J., and Mas, P. (2014). The impact of chromatin dynamics on plant light responses and circadian clock function. J. Exp. Bot. 65, 28952913. doi: 10.1093/jxb/eru011

Baroux, C., Pecinka, A., Fuchs, J., Schubert, I., and Grossniklaus, U. (2007). The triploid endosperm genome of arabidopsis adopts a peculiar, parental-dosagedependent chromatin organization. Plant Cell 19, 1782. doi: 10.1105/ tpc.106.046235

Benhamed, M., Bertrand, C., Servet, C., and Zhou, D. X. (2006). Arabidopsis GCN5, HD1, and TAF1/HAF2 interact to regulate histone acetylation required for light-responsive gene expression. Plant Cell 18, 2893-2903. doi: 10.1105/ tpc.106.043489

Benvenuto, G., Formiggini, F., Laflamme, P., Malakhov, M., and Bowler, C. (2002). The photomorphogenesis regulator DET1 binds the amino-terminal tail of histone H2B in a nucleosome context. Curr. Biol. 12, 1529-1534. doi: 10.1016/ S0960-9822(02)01105-3

Berger, S. L. (2000). Local or global? Nature 408, 412-415. doi: 10.1038/35044160

Bi, X., Cheng, Y.-J., Hu, B., Ma, X., Wu, R., Wang, J.-W., et al. (2017). Nonrandom domain organization of the Arabidopsis genome at the nuclear periphery. Genome Res. 27, 1162-1173. doi: 10.1101/gr.215186.116

Bickmore, W. A., and van Steensel, B. (2013). Genome architecture: domain organization of interphase chromosomes. Cell 152, 1270-1284. doi: 10.1016/ j.cell.2013.02.001 responses though priming mechanisms (transcriptional memory) or through trans-generational inheritance of UV-regulated epigenetic processes remain poorly investigated as compared to other plant stresses (Müller-Xing et al., 2014).

\section{AUTHOR CONTRIBUTIONS}

All authors contributed to writing and funding research activity.

\section{FUNDING}

This work is supported by the EPIPLANT Groupement de Recherche (France). Work by $\mathrm{CB}$ and FB is supported by the Velux Foundation (Switzerland), MEMOLIFE (ANR-10-LABX54), and PSL ${ }^{\star}$ Research University (ANR-11-IDEX-0001-02). Work by FB is supported by the French National Research Agency grants Chromalight (ANR-18-CE13-0004-01) and RiboStress (ANR-17-CE12-0026-02), and the COST Action CA16212 INDEPTH (E.U.). Work by CL is supported by the French National Research Agency (ANR-14-CE02-0010).

\section{ACKNOWLEDGMENTS}

The authors acknowledge Chris Bowler (IBENS, Paris, France) for critical reading of the manuscript and for constant support. We also apologize for not citing all the relevant primary research articles and reviews due to space limitations.

Binkert, M., Crocco, C. D., Ekundayo, B., Lau, K., Raffelberg, S., Tilbrook, K., et al. (2016). Revisiting chromatin binding of the Arabidopsis UV-B photoreceptor UVR8. BMC Plant Biol. 16, 42. doi: 10.1186/s12870-016-0732-5

Bourbousse, C., Ahmed, I., Roudier, F., Zabulon, G., Blondet, E., Balzergue, S., et al. (2012). Histone $\mathrm{H} 2 \mathrm{~B}$ monoubiquitination facilitates the rapid modulation of gene expression during arabidopsis photomorphogenesis. PloS Genet. 8, e1002825. doi: 10.1371/journal.pgen.1002825

Bourbousse, C., Mestiri, I., Zabulon, G., Bourge, M., Formiggini, F., Koini, M. A., et al. (2015). Light signaling controls nuclear architecture reorganization during seedling establishment. Proc. Natl. Acad. Sci. U.S.A. 112, E2836. doi: 10.1073/pnas. 1503512112

Brown, B. A., Cloix, C., Jiang, G. H., Kaiserli, E., Herzyk, P., Kliebenstein, D. J., et al. (2005). A UV-B-specific signaling component orchestrates plant UV protection. Proc. Natl. Acad. Sci. U.S.A. 102, 18225. doi: 10.1073/ pnas.0507187102

Casal, J. J. (2013). Photoreceptor signaling networks in plant responses to shade. Annu. Rev. Plant Biol. 64, 403-427. doi: 10.1146/annurev-arplant-050312120221

Chan, K. X., Phua, S. Y., Crisp, P., McQuinn, R., and Pogson, B. J. (2016). Learning the languages of the chloroplast: retrograde signaling and beyond. Annu. Rev. Plant Biol. 67, 25-53. doi: 10.1146/annurev-arplant-043015-111854

Charron, J.-B. F., He, H., Elling, A. A., and Deng, X. W. (2009). Dynamic landscapes of four histone modifications during deetiolation in Arabidopsis. Plant Cell Online 21, 3732-3748. doi: 10.1105/tpc.109.066845

Chen, F., Li, B., Li, G., Charron, J.-B., Dai, M., Shi, X., et al. (2014). Arabidopsis phytochrome a directly targets numerous promoters for individualized modulation of genes in a wide range of pathways. Plant Cell 26, 1949. doi: 10.1105/tpc.114.123950 
Chory, J. (2010). Light signal transduction: an infinite spectrum of possibilities. Plant J. 61, 982-991. doi: 10.1111/j.1365-313X.2009.04105.x

Chua, Y. L., Brown, A. P. C., and Gray, J. C. (2001). Targeted histone acetylation and altered nuclease accessibility over short regions of the pea plastocyanin gene. Plant Cell 13, 599-612. doi: 10.2307/3871409

Chua, Y. L., Watson, L. A., and Gray, J. C. (2003). The transcriptional enhancer of the pea plastocyanin gene associates with the nuclear matrix and regulates gene expression through histone acetylation. Plant Cell 15, 1468-1479. doi: 10.1105/ tpc. 011825

Cloix, C., and Jenkins, G. I. (2008). Interaction of the Arabidopsis UV-B-specific signaling component UVR8 with chromatin. Mol. Plant 1, 118-128. doi: $10.1093 / \mathrm{mp} / \mathrm{ssm} 012$

Cookson, S. J., RADZIEJWOSKI, A., and GRANIER, C. (2006). Cell and leaf size plasticity in Arabidopsis: what is the role of endoreduplication? Plant Cell Environ. 29, 1273-1283. doi: 10.1111/j.1365-3040.2006.01506.x

Croce, R., and van Amerongen, H. (2014). Natural strategies for photosynthetic light harvesting. Nat. Chem. Biol. 10, 492. doi: 10.1038/nchembio.1555

Cutler, S. R., Ehrhardt, D. W., Griffitts, J. S., and Somerville, C. R. (2000). Random GFP::cDNA fusions enable visualization of subcellular structures in cells of Arabidopsis at a high frequency. Proc. Natl. Acad. Sci. U.S.A. 97, 3718. doi: 10.1073/pnas.97.7.3718

Demarsy, E., Goldschmidt-Clermont, M., and Ulm, R. (2018). Coping with 'Dark Sides of the Sun' through photoreceptor signaling. Trends Plant Sci. 23, 260271. doi: $10.1016 /$ j.tplants.2017.11.007

Donà, M., and Mittelsten Scheid, O. (2015). DNA damage repair in the context of plant chromatin. Plant Physiol. 168, 1206. doi: 10.1104/pp.15.00538

Dong, J., Tang, D., Gao, Z., Yu, R., Li, K., He, H., et al. (2014). Arabidopsis DEETIOLATED1 represses photomorphogenesis by positively regulating phytochrome-interacting factors in the dark. Plant Cell 26, 3630-3645. doi: 10.1105/tpc.114.130666

Douet, J., Blanchard, B., Cuvillier, C., and Tourmente, S. (2008). Interplay of RNA Pol IV and ROS1 during post-embryonic 5S rDNA chromatin remodeling. Plant Cell Physiol. 49, 1783-1791. doi: 10.1093/pcp/pcn152

Doyle, J. J., and Coate, J. E. (2018). Polyploidy, the nucleotype, and novelty: the impact of genome doubling on the biology of the cell. Int. J. Plant Sci. 180, 152. doi: $10.1086 / 700636$

Duarte-Aké, F. (2019). Epigenetic changes and photosynthetic plasticity in response to environment. Environ. Exp. Bot. v. 159, 108-120. doi: 10.1016/ j.envexpbot.2018.12.010

Eberhard, S., Finazzi, G., and Wollman, F. A. (2008). The dynamics of photosynthesis. Annu. Rev. Genet. 42, 463-515. doi: 10.1146/ annurev.genet.42.110807.091452

Favory, J., Stec, A., Gruber, H., Rizzini, L., Oravecz, A., Funk, M., et al. (2009). Interaction of COP1 and UVR8 regulates UV-B-induced photomorphogenesis and stress acclimation in Arabidopsis. EMBO J. 28, 591. doi: 10.1038/emboj.2009.4

Feng, C.-M., Qiu, Y., Van Buskirk, E. K., Yang, E. J., and Chen, M. (2014a). Lightregulated gene repositioning in Arabidopsis. Nat. Commun. 5, 3027. doi: $10.1038 /$ ncomms 4027

Feng, S., Cokus, S. J., Schubert, V., Zhai, J., Pellegrini, M., and Jacobsen, S. E. (2014b). Genome-wide Hi-C analyses in wild-type and mutants reveal highresolution chromatin interactions in Arabidopsis. Mol. Cell 55, 694-707. doi: 10.1016/j.molcel.2014.07.008

Fina, J. P., Masotti, F., Rius, S. P., Crevacuore, F., and Casati, P. (2017). HAC1 and HAF1 histone acetyltransferases have different roles in UV-B responses in Arabidopsis. Front. Plant Sci. 8, 1179. doi: 10.3389/fpls.2017.01179

Fondriest Environmental, Inc. (2014). Solar Radiation and Photosynethically Active Radiation. Available at: https://www.fondriest.com/environmentalmeasurements/parameters/weather/photosynthetically-active-radiation/.

Fonseca, S., and Rubio, V. (2019). Arabidopsis CRL4 complexes: surveying chromatin states and gene expression. Front. Plant Sci. 10, 1095. doi: 10.3389/fpls.2019.01095

Fransz, P., de Jong, J. H., Lysak, M., Castiglione, M. R., and Schubert, I. (2002). Interphase chromosomes in Arabidopsis are organized as well defined chromocenters from which euchromatin loops emanate. Proc. Natl. Acad. Sci. U.S.A. 99, 14584. doi: 10.1073/pnas.212325299

Galvão, V. C., and Fankhauser, C. (2015). Sensing the light environment in plants: photoreceptors and early signaling steps. Curr. Opin. Neurobiol. 34, 46-53. doi: 10.1016/j.conb.2015.01.013
Gegas, V. C., Wargent, J. J., Pesquet, E., Granqvist, E., Paul, N. D., and Doonan, J. H. (2014). Endopolyploidy as a potential alternative adaptive strategy for Arabidopsis leaf size variation in response to UV-B. J. Exp. Bot. 65, 2757-2766. doi: $10.1093 / \mathrm{jxb} / \mathrm{ert} 473$

Gendreau, E., Traas, J., Desnos, T., Grandjean, O., Caboche, M., and Hofte, H. (1997). Cellular basis of hypocotyl growth in Arabidopsis thaliana. Plant Physiol. 114, 295-305. doi: 10.1104/pp.114.1.295

Godoy Herz, M. A., Kubaczka, M. G., Brzyżek, G., Servi, L., Krzyszton, M., Simpson, C., et al. (2019). Light regulates plant alternative splicing through the control of transcriptional elongation. Mol. Cell 73, 1066-1074.e3. doi: 10.1016/ j.molcel.2018.12.005

Graindorge, S., Cognat, V., Johann to Berens, P., Mutterer, J., and Molinier, J. (2019). Photodamage repair pathways contribute to the accurate maintenance of the DNA methylome landscape upon UV exposure. PloS Genet. 15, e1008476. doi: 10.1371/journal.pgen.1008476

Guo, H., Yang, H., Mockler, T. C., and Lin, C. (1998). Regulation of flowering time by Arabidopsis photoreceptors. Science 279, 1360-1363. doi: 10.1126/ science. 279.5355 .1360

Han, S.-K., Wu, M.-F., Cui, S., and Wagner, D. (2015). Roles and activities of chromatin remodeling ATPases in plants. Plant J. 83, 62-77. doi: 10.1111/ tpj. 12877

He, S., Vickers, M., Zhang, J., and Feng, X. (2019). Natural depletion of histone H1 in sex cells causes DNA demethylation, heterochromatin decondensation and transposon activation. eLife 8, e42530. doi: 10.7554/eLife.42530

Henry, K. W., Wyce, A., Lo, W. S., Duggan, L. J., Emre, N. C. T., Kao, C. F., et al. (2003). Transcriptional activation via sequential histone H2B ubiquitylation and deubiquitylation, mediated by SAGA-associated Ubp8. Genes Dev. 17, 2648-2663. doi: 10.1101/gad.1144003

Jégu, T., Veluchamy, A., Ramirez-Prado, J. S., Rizzi-Paillet, C., Perez, M., Lhomme, A., et al. (2017). The Arabidopsis SWI/SNF protein BAF60 mediates seedling growth control by modulating DNA accessibility. Genome Biol. 18, 114. doi: 10.1186/s13059-017-1246-7

Jenkins, G. I. (2017). Photomorphogenic responses to ultraviolet-B light. Plant Cell Environ. 40, 2544-2557. doi: 10.1111/pce.12934

Jeusset, M. L., and McManus, J. K. (2019). Developing targeted therapies that exploit aberrant histone ubiquitination in cancer. Cells 8, 165. doi: 10.3390/ cells 8020165

Jing, Y., Zhang, D., Wang, X., Tang, W., Wang, W., Huai, J., et al. (2013). Arabidopsis chromatin remodeling factor PICKLE interacts with transcription factor HY5 to regulate hypocotyl cell elongation. Plant Cell 25, 242. doi: 10.1105/tpc.112.105742

Jovtchev, G., Schubert, V., Meister, A., Barow, M., and Schubert, I. (2006). Nuclear DNA content and nuclear and cell volume are positively correlated in angiosperms. Cytogenet. Genome Res. 114, 77-82. doi: 10.1159/000091932

Jung, J.-H., Domijan, M., Klose, C., Biswas, S., Ezer, D., Gao, M., et al. (2016). Phytochromes function as thermosensors in Arabidopsis. Science 354, 886. doi: 10.1126/science.aaf6005

Kaiserli, E., Perrella, G., and Davidson, M. L. (2018). Light and temperature shape nuclear architecture and gene expression. Curr. Opin. Plant Biol. 45, 103-111. doi: 10.1016/j.pbi.2018.05.018

Kami, C., Lorrain, S., Hornitschek, P., and Fankhauser, C. (2010). "Chapter Two Light-Regulated Plant Growth and Development," in Current Topics in Developmental Biology. Ed. M. C. P. Timmermans (Academic Press), 29-66. doi: $10.1016 /$ S0070-2153(10)91002-8

Kiel, C., Yus, E., and Serrano, L. (2010). Engineering signal transduction pathways. Cell 140, 33-47. doi: 10.1016/j.cell.2009.12.028

Kimura, S., and Sakaguchi, K. (2006). DNA repair in plants. Chem. Rev. 106, 753766. doi: $10.1021 / \mathrm{cr} 040482 \mathrm{n}$

Kinoshita, I., Sanbe, A., and Yokomura, E. (2008). Difference in light-induced increase in ploidy level and cell size between adaxial and abaxial epidermal pavement cells of Phaseolus vulgaris primary leaves. J. Exp. Bot. 59, 1419-1430. doi: $10.1093 / \mathrm{jxb} / \mathrm{ern} 055$

Kirik, V., Schrader, A., Uhrig, J. F., and Hulskamp, M. (2007). MIDGET unravels functions of the arabidopsis topoisomerase VI complex in DNA endoreduplication, chromatin condensation, and transcriptional silencing. Plant Cell 19, 3100. doi: 10.1105/tpc.107.054361

Lau, O. S., Huang, X., Charron, J.-B., Lee, J.-H., Li, G., and Deng, X. W. (2011). Interaction of Arabidopsis DET1 with CCA1 and LHY in mediating 
transcriptional repression in the plant circadian clock. Mol. Cell 43, 703-712. doi: 10.1016/j.molcel.2011.07.013

Leivar, P., and Quail, P. H. (2011). PIFs: pivotal components in a cellular signaling hub. Trends Plant Sci. 16, 19-28. doi: 10.1016/j.tplants.2010.08.003

Li, J., Terzaghi, W., and Deng, X. W. (2012). Genomic basis for light control of plant development. Protein Cell 3, 106-116. doi: 10.1007/s13238-012-2016-7

Li, Y., Mukherjee, I., Thum, K. E., Tanurdzic, M., Katari, M. S., Obertello, M., et al. (2015). The histone methyltransferase SDG8 mediates the epigenetic modification of light and carbon responsive genes in plants. Genome Biol. 16, 79. doi: 10.1186/s13059-015-0640-2

Li, Z., Wakao, S., Fischer, B. B., and Niyogi, K. K. (2009). Sensing and responding to excess light. Annu. Rev. Plant Biol. 60, 239-260. doi: 10.1146/ annurev.arplant.58.032806.103844

Liang, T., Mei, S., Shi, C., Yang, Y., Peng, Y., Ma, L., et al. (2018). UVR8 interacts with BES1 and BIM1 to regulate transcription and photomorphogenesis in Arabidopsis. Dev. Cell 44, 512-523.e5. doi: 10.1016/j.devcel.2017.12.028

Liu, H., Yu, X., Li, K., Klejnot, J., Yang, H., Lisiero, D., et al. (2008). Photoexcited CRY2 interacts with CIB1 to regulate transcription and floral initiation in Arabidopsis. Science 322, 1535-1539. doi: 10.1126/science.1163927

Liu, X., Chen, C.-Y., Wang, K.-C., Luo, M., Tai, R., Yuan, L., et al. (2013). Phytochrome interacting Factor3 Associates with the Histone Deacetylase HDA15 in repression of chlorophyll biosynthesis and photosynthesis in etiolated Arabidopsis seedlings. Plant Cell 25, 1258. doi: 10.1105/tpc.113.109710

Liu, X., Wei, W., Zhu, W., Su, L., Xiong, Z., Zhou, M., et al. (2017a). Histone Deacetylase AtSRT1 links metabolic flux and stress response in arabidopsis. Mol. Plant 10, 1510-1522. doi: 10.1016/j.molp.2017.10.010

Liu, Y., Zhang, W., Zhang, K., You, Q., Yan, H., Jiao, Y., et al. (2017b). Genomewide mapping of DNase I hypersensitive sites reveals chromatin accessibility changes in Arabidopsis euchromatin and heterochromatin regions under extended darkness. Sci. Rep. 7, 4093. doi: 10.1038/s41598-017-04524-9

Lopez-Juez, E., Dillon, E., Magyar, Z., Khan, S., Hazeldine, S., de Jager, S. M., et al. (2008). Distinct light-initiated gene expression and cell cycle programs in the shoot apex and cotyledons of Arabidopsis. Plant Cell 20, 947-968. doi: 10.1105/ tpc.107.057075

Ma, D., Li, X., Guo, Y., Chu, J., Fang, S., Yan, C., et al. (2016). Cryptochrome 1 interacts with PIF4 to regulate high temperature-mediated hypocotyl elongation in response to blue light. Proc. Natl. Acad. Sci. U.S.A. 113, 224. doi: $10.1073 /$ pnas.1511437113

Mathieu, O., Jasencakova, Z., Vaillant, I., Gendrel, A.-V., Colot, V., Schubert, I., et al. (2003). Changes in $5 \mathrm{~S}$ rDNA chromatin organization and transcription during heterochromatin establishment in Arabidopsis 10.1105/tpc.017467. Plant Cell Online 15, 2929-2939. doi: 10.1105/tpc.017467

Maxwell, B. B., Andersson, C. R., Poole, D. S., Kay, S. A., and Chory, J. (2003). HY5, Circadian Clock-Associated 1, and a cis-element, DET1 dark response element, mediate DET1 regulation of chlorophyll a/b-binding protein 2 expression. Plant Physiol. 133, 1565-1577. doi: 10.1104/pp.103.025114

Molinier, J. (2017). Genome and epigenome surveillance processes underlying UV exposure in plants. Genes 8, 316. doi: 10.3390/genes8110316

Müller-Xing, R., Xing, Q., and Goodrich, J. (2014). Footprints of the sun: memory of UV and light stress in plants. Front. Plant Sci. 5, 474. doi: 10.3389/ fpls.2014.00474

Nassrallah, A., Rougée, M., Bourbousse, C., Drevensek, S., Fonseca, S., Iniesto, E., et al. (2018). DET1-mediated degradation of a SAGA-like deubiquitination module controls H2Bub homeostasis. eL ife 7, e37892. doi: 10.7554/eLife.37892

Ni, M., Tepperman, J. M., and Quail, P. H. (1998). PIF3, a phytochromeinteracting factor necessary for normal photoinduced signal transduction, is a novel basic helix-loop-helix protein. Cell 95, 657-667. doi: 10.1016/s00928674(00)81636-0

Osterlund, M. T., Hardtke, C. S., Wei, N., and Deng, X. W. (2000). Targeted destabilization of HY5 during light-regulated development of Arabidopsis. Nature 405, 462-466. doi: 10.1038/35013076

Pass, D. A., Sornay, E., Marchbank, A., Crawford, M. R., Paszkiewicz, K., Kent, N. A., et al. (2017). Genome-wide chromatin mapping with size resolution reveals a dynamic sub-nucleosomal landscape in Arabidopsis. PloS Genet. 13, e1006988. doi: 10.1371/journal.pgen.1006988

Pedmale, U. V., Huang, S. C., Zander, M., Cole, B. J., Hetzel, J., Ljung, K., et al. (2016). Cryptochromes interact directly with PIFs to control plant growth in limiting blue light. Cell 164, 233-245. doi: 10.1016/j.cell.2015.12.018
Perissi, V., and Rosenfeld, M. G. (2005). Controlling nuclear receptors: the circular logic of cofactor cycles. Nat. Rev. Mol. Cell Biol. 6, 542-554. doi: 10.1038/ nrm 1680

Perrella, G., and Kaiserli, E. (2016). Light behind the curtain: photoregulation of nuclear architecture and chromatin dynamics in plants. New Phytol. 212, 908919. doi: $10.1111 /$ nph. 14269

Poulet, A., Duc, C., Voisin, M., Desset, S., Tutois, S., Vanrobays, E., et al. (2017). The LINC complex contributes to heterochromatin organisation and transcriptional gene silencing in plants. J. Cell Sci. 130, 590. doi: 10.1242/jcs. 194712

Probst, A. V., and Almouzni, G. (2011). Heterochromatin establishment in the context of genome-wide epigenetic reprogramming. Trends Genet. 27, 177185. doi: $10.1016 /$ j.tig.2011.02.002

Rochaix, J.-D. (2014). Regulation and dynamics of the light-harvesting system. Annu. Rev. Plant Biol. 65, 287-309. doi: 10.1146/annurev-arplant-050213-040226

Rutowicz, K., Puzio, M., Halibart-Puzio, J., Lirski, M., Kroten, M. A., Kotlinski, M., et al. (2015). A specialized histone H1 variant is required for adaptive responses to complex abiotic stress and related DNA methylation in Arabidopsis. Plant Physiol. 169, 2080. doi: 10.1104/pp.15.00493

Sage, L. C. (Eds.) (1992). "Index," in Pigment of the Imagination (Boston: Academic Press), 547-562. doi: 10.1016/B978-0-12-614445-1.50039-1

Schubert, V., Berr, A., and Meister, A. (2012). Interphase chromatin organisation in Arabidopsis nuclei: constraints versus randomness. Chromosoma 121, 369387. doi: $10.1007 / \mathrm{s} 00412-012-0367-8$

Schuster-Böckler, B., and Lehner, B. (2012). Chromatin organization is a major influence on regional mutation rates in human cancer cells. Nature 488, 504. doi: 10.1038 /nature 11273

Seluzicki, A., Burko, Y., and Chory, J. (2017). Dancing in the dark: darkness as a signal in plants. Plant Cell Environ. 40, 2487-2501. doi: 10.1111/pce.12900

She, W., Grimanelli, D., Rutowicz, K., Whitehead, M. W. J., Puzio, M., Kotliński, M., et al. (2013). Chromatin reprogramming during the somatic-toreproductive cell fate transition in plants. Development 140, 4008. doi: 10.1242/dev.095034

Šimková, K., Moreau, F., Pawlak, P., Vriet, C., Baruah, A., Alexandre, C., et al. (2012). Integration of stress-related and reactive oxygen species-mediated signals by Topoisomerase VI in Arabidopsis thaliana. Proc. Natl. Acad. Sci. U.S.A. 109, 16360. doi: 10.1073/pnas.1202041109

Simon, L., Voisin, M., Tatout, C., and Probst, A. V. (2015). Structure and Function of Centromeric and Pericentromeric Heterochromatin in Arabidopsis thaliana. Front. Plant Sci. 6, 1049. doi: 10.3389/fpls.2015.01049

Smith, K. S., Liu, L. L., Ganesan, S., Michor, F., and De, S. (2017). Nuclear topology modulates the mutational landscapes of cancer genomes. Nat. Struct. Mol. Biol. 24, 1000. doi: 10.1038/nsmb.3474

Snoek, B. L., Pavlova, P., Tessadori, F., Peeters, A. J. M., Bourbousse, C., Barneche, F., et al. (2017). Genetic dissection of morphometric traits reveals that phytochrome $\mathrm{B}$ affects nucleus size and heterochromatin organization in Arabidopsis thaliana. (Bethesda) 7, 2519-2531. doi: 10.1534/g3.117.043539

Solovei, I., Kreysing, M., Lanctôt, C., Kösem, S., Peichl, L., Cremer, T., et al. (2009). Nuclear architecture of rod photoreceptor cells adapts to vision in mammalian evolution. Cell 137, 356-368. doi: 10.1016/j.cell.2009.01.052

Solovei, I., Wang, A. S., Thanisch, K., Schmidt, C. S., Krebs, S., Zwerger, M., et al. (2013). LBR and lamin A/C sequentially tether peripheral heterochromatin and inversely regulate differentiation. Cell 152, 584-598. doi: 10.1016/ j.cell.2013.01.009

Suganuma, T., and Workman, J. L. (2018). Chromatin and metabolism. Annu. Rev. Biochem. 87, 27-49. doi: 10.1146/annurev-biochem-062917-012634

Sullivan, A. M., Arsovski, A. A., Lempe, J., Bubb, K. L., Weirauch, M. T., Sabo, P. J., et al. (2014). Mapping and dynamics of regulatory DNA and transcription factor networks in A. thaliana. Cell Rep. 8, 2015-2030. doi: 10.1016/ j.celrep.2014.08.019

Takata, H., Hanafusa, T., Mori, T., Shimura, M., Iida, Y., Ishikawa, K., et al. (2013). Chromatin compaction protects genomic DNA from radiation damage. PloS One 8, e75622. doi: 10.1371/journal.pone.0075622

Tang, Y., Liu, X., Liu, X., Li, Y., Wu, K., and Hou, X. (2017). Arabidopsis NF-YCs mediate the light-controlled Hypocotyl Elongation via modulating histone acetylation. Mol. Plant 10, 260-273. doi: 10.1016/j.molp.2016.11.007

Tessadori, F., Schulkes, R. K., van Driel, R., and Fransz, P. (2007). Light-regulated large-scale reorganization of chromatin during the floral transition in Arabidopsis. Plant J. 50, 848-857. doi: 10.1111/j.1365-313X.2007.03093.x 
Tessadori, F., van Zanten, M., Pavlova, P., Clifton, R., Pontvianne, F., Snoek, L. B., et al. (2009). Phytochrome B and Histone Deacetylase 6 control light-induced chromatin compaction in Arabidopsis thaliana. PloS Genet. 5, e1000638. doi: 10.1371/journal.pgen.1000638

Van Buskirk, E. K., Decker, P. V., and Chen, M. (2012). Photobodies in light signaling. Plant Physiol. 158, 52. doi: 10.1104/pp.111.186411

van Zanten, M., Tessadori, F., Bossen, L., Peeters, A. J. M., and Fransz, P. (2010a). Large-scale chromatin de-compaction induced by low light is not accompanied by nucleosomal displacement. Plant Signaling Behav. 5, 1677-1678. doi: 10.4161/psb.5.12.14039

van Zanten, M., Tessadori, F., McLoughlin, F., Smith, R., Millenaar, F. F., van Driel, R., et al. (2010b). Photoreceptors CRYTOCHROME2 and phytochrome B control chromatin compaction in Arabidopsis. Plant Physiol. 154, 1686. doi: 10.1104/pp.110.164616

van Zanten, M., Koini, M. A., Geyer, R., Liu, Y., Brambilla, V., Bartels, D., et al. (2011). Seed maturation in Arabidopsis thaliana is characterized by nuclear size reduction and increased chromatin condensation. Proc. Natl. Acad. Sci. U.S.A. 108, 20219-20224. doi: 10.1073/pnas.1117726108

van Zanten, M., Tessadori, F., Peeters, A. J. M., and Fransz, P. (2012). Shedding light on large-scale chromatin reorganization in Arabidopsis thaliana. Mol. Plant 5, 583-590. doi: 10.1093/mp/sss030

Willing, E.-M., Piofczyk, T., Albert, A., Winkler, J. B., Schneeberger, K., and Pecinka, A. (2016). UVR2 ensures transgenerational genome stability under simulated natural UV-B in Arabidopsis thaliana. Nat. Commun. 7, 13522. doi: $10.1038 /$ ncomms 13522

Xiao, J., Jin, R., and Wagner, D. (2017). Developmental transitions: integrating environmental cues with hormonal signaling in the chromatin landscape in plants. Genome Biol. 18, 88. doi: 10.1186/s13059-017-1228-9

Yang, L., Mo, W., Yu, X., Yao, N., Zhou, Z., Fan, X., et al. (2018). Reconstituting Arabidopsis CRY2 signaling pathway in Mammalian cells reveals regulation of transcription by direct binding of CRY2 to DNA. Cell Rep. 24, 585-593.e4. doi: 10.1016/j.celrep.2018.06.069

Yin, R., and Ulm, R. (2017). How plants cope with UV-B: from perception to response. Curr. Opin. Plant Biol. 37, 42-48. doi: 10.1016/j.pbi.2017.03.013

Yin, Y., Cheong, H., Friedrichsen, D., Zhao, Y., Hu, J., Mora-Garcia, S., et al. (2002). A crucial role for the putative Arabidopsis topoisomerase VI in plant growth and development. Proc. Natl. Acad. Sci. U.S.A. 99, 10191. doi: 10.1073/ pnas.152337599

Zhang, D., Jing, Y., Jiang, Z., and Lin, R. (2014). The chromatin-remodeling factor PICKLE integrates Brassinosteroid and gibberellin signaling during skotomorphogenic growth in Arabidopsis. Plant Cell 26, 2472. doi: 10.1105/ tpc. 113.121848

Zhang, D., Li, Y., Zhang, X., Zha, P., and Lin, R. (2017). The SWI2/SNF2 chromatin-remodeling ATPase BRAHMA regulates Chlorophyll biosynthesis in arabidopsis. Mol. Plant 10, 155-167. doi: 10.1016/j.molp.2016.11.003

Zhao, L., Peng, T., Chen, C.-Y., Ji, R., Gu, D., Li, T., et al. (2019). HY5 interacts with the histone deacetylase HDA15 to repress hypocotyl cell elongation in photomorphogenesis. Plant Physiol. 180, 1450. doi: 10.1104/pp.19.00055

Conflict of Interest: The authors declare that the research was conducted in the absence of any commercial or financial relationships that could be construed as a potential conflict of interest.

Copyright (c) 2020 Bourbousse, Barneche and Laloi. This is an open-access article distributed under the terms of the Creative Commons Attribution License (CC BY). The use, distribution or reproduction in other forums is permitted, provided the original author(s) and the copyright owner(s) are credited and that the original publication in this journal is cited, in accordance with accepted academic practice. No use, distribution or reproduction is permitted which does not comply with these terms. 